\title{
Biomimetics: networking to turn scientific knowledge into beneficial practice
}

\author{
R. W. Erb \\ BIOKON International - The Biomimetics Association, Berlin, Germany
}

\begin{abstract}
$B I O K O N$ International is a non-profit organization and focuses on international initiatives and measures to build an integrative network of leading experts in the field of biomimetics. The primary objective is to make biomimetics useful as a source of ideas and innovations for technology, business, and society. The association combines resources and competencies in the area of biomimetics, bionics and bio-inspired technologies to achieve sustained success. By sharing our knowledge, biomimetics products and technologies as drivers of innovation are promoted.

The presentation focuses on innovation management and how biomimetics networks contribute to turn scientific knowledge into beneficial practice.

Keywords: biomimetics, network, innovation, knowledge transfer, interdisciplinarity.
\end{abstract}

\section{Biomimetics - interdisciplinarity and networking}

Biomimetics is based on the interdisciplinary link that extends from fundamental research to multi-faceted high-tech applications in and with a previously unknown scope and reach. It relies on interaction between biological and technical expertise with a clearly application-orientated approach. Successful transfer of knowledge concerning the structures, processes and characteristics of living systems to technical systems and their application in industrial practice is a "conditio sine qua non" for intelligible communication, correspondence and cooperation between disciplines.

The complexity of today's challenges means that a single discipline is unable to face them alone. An interdisciplinary dialogue is vital in coping with these challenges, and precisely, this is one of biomimetics' strongest points. 


\section{Networked structures as a criterion for success}

Nature is one of the most successful innovators of all time! For some 3.8 billion years, nature has developed, tested and optimised new solutions to problems time and time again. Biomimetics plays a key role whenever humans seek to leverage solutions successfully tested in nature's test lab for our knowledge society. This is why it is not just a melting pot of different biological, scientific and engineering disciplines, but a field of research in which fundamental research and technical applicability mesh at a very early stage.

Biomimetics combine biology and technology. It focuses on tried-and-trusted natural solutions and their systematic implementation in technology [1]. Scientists and engineers from various fields collaborate in an interdisciplinary approach to generate and launch innovative ideas and developments. And precisely this is a golden opportunity for businesses. Afterall, biomimetics deliver innovations. Our ambitious goal is often that of developing new concepts and products that extend well beyond what appears to be technologically feasible today. This is why biomimetics is gaining in popularity and increasingly being adopted in industrial research and development.

Biomimetics ideas and concepts play a role in many development and optimisation processes and are increasingly finding their way into the final product - although the contribution made by biomimetics is not always explicitly mentioned. For example, when Opel achieves a new standard with respect to lightweight construction and structural rigidity with a biomimetically optimised stub axle, this does not mean that the car will be promoted as a biomimetics product. This also applies to product contributions in other fields: aircraft wings, MP3s, Velcro fasteners - all of which are established products for which nature provided the role models. There is one important thing to note here: it is not just a case of copying nature, but of discovering the underlying principles and methods, and converting them into tangible added value for technology, business and society - and this necessitates interdisciplinary networking and cooperation.

\section{BIOKON - biomimetics network of excellence}

The biomimetics network of excellence, BIOKON, was founded in 2001 with support from the German Federal Ministry of Education and Research (BMBF). In 2004, BIOKON adopted the legal status of a non-profit association. BIOKON is the umbrella for over 80 universities, research institutions, businesses and individuals from the whole of Germany and Europe, all of whom work in the field of biomimetics [2].

The major aims of $B I O K O N$ are: bundling and networking existing expert knowledge, implementing or accompanying R\&D projects, training and educational efforts, and offering and coordinating PR for bionics. BIOKON converts knowledge into added value and acts as a lobbyist and provider of impetus. In recent years, $B I O K O N$ has established itself as a central hub for information on and contacts in bionics and thus developed into a peer-level liaison 
entity for various user groups or user industries. $B I O K O N$ has become a "drive belt for patented, natural solutions".

The biomimetics network of excellence is founded on a growing number of regional nodes which are distributed throughout Germany and research into various sub-disciplines in the field of biomimetics. The members of BIOKON in turn liaise with leading research institutions all over the world. Many industrial enterprises in Germany, and increasingly abroad, already draw on the competency pool offered by $B I O K O N$, adopting biomimetics ideas, using expert consultancy, involving $B I O K O N$ experts in their R\&D work, or commissioning tangible research projects. Some major companies have created their own liaison interfaces for cooperation with $B I O K O N$; small to medium sized enterprises benefit from the research fed to them by universities and colleges and have been able to assert themselves more easily on today's markets thanks to the rapid implementation of tangible product ideas and biomimetics methods. Enquiries from companies in turn generate new bionic topics that lead to a stronger focus of fundamental biological research on application-relevant questions.

Besides BIOKON, there are local networks in Germany, such as the Biomimetics Competency Network based in Baden Wuerttemberg, the Bionic Innovation Centre (B-I-C) at Bremen University, or the bionic engineering network (BEN) in Saarland. All of them are integrated vertically with the national structures and pillars of these structures at a regional level.

BIOKON has collaborated with the German Engineering Association (Verein Deutscher Ingenieure (VDI)) and the German Federal Ministries of Education and Research (BMBF), Economics and Technology (BMWi) and the Environment (BMU), as well as the German government's national liaison offices for the European framework research programme to develop the technology roadmap "Bionik 2020". In the field of biomimetics, Germany is in the lucky position of being one of the most prominent research locations worldwide. Maintaining the high scientific level of German research in a country with little in the line of raw materials will continue to be a major concern in future. To allow this to happen, it is imperative to further reinforce the international reputation and integrate biomimetics approaches permanently and rapidly with industrial innovation processes. In particular, this means intensifying knowledge transfer to industry. This explains why transfer of knowhow is so firmly in the focus of the biomimetics roadmap.

\section{Internationalisation: $B I O K O N$ International: The Biomimetics Association}

The national networking structures created by $B I O K O N$ are a genuine success story. At the same time, wide reaching international contacts and working relationships, especially to European partners, have been established in recent years. The foundation of the international umbrella organisation BIOKON International - The Biomimetics Association in March 2009 was the next logical step towards international networking and institutionalisation. The registered 
office of this umbrella organisation for national bionics networks is Berlin; the offices are run by $B I O K O N$.

Shortly after its foundation, $B I O K O N$ International already has 80 members (member institutes) from 11 different countries and acts as a recognised stakeholder on an international level. Its major activities focus on developing mutual R\&D projects, generating and implementing financing options for biomimetics research and development at an international level, and ultimately on firmly establishing biomimetics as a driver of innovation for new products and technologies on a European and international platform [3].

\section{Networks as a driver of innovation}

Biomimetics networks bundle the expertise from various fields of biological, natural and engineering sciences - and increasingly from business - in a versatile manner and at many levels. Biomimetics solutions and optimisation strategies provide an increasingly targeted approach to novel products and technologies and have proved their superiority to competitive solutions. Another characteristic attributable to biomimetics developments is that they do not just lead to a single product line, but to a wide ranging product portfolio that often spans many branches of industry. Accompanying the prime example of biomimetics, the Lotus Effect $^{\circledR}$, a seminal innovation that is one of the 12 most significant innovations to have come from Germany in the last fifty years, there are now 200 auxiliary innovations. Other examples besides the Lotus Effect ${ }^{\circledR}$ include the use of the optimisation programs by Professor Mattheck and Professor Rechenberg, and more recently the Fin Ray Effect ${ }^{\circledR}$ by EvoLogics, of which the same can be said.

However, the path from a biomimetics idea to a marketable product is typically a long one. Decisive steps along this path include manufacturing of technical prototypes and functional models, close collaboration on mutual R\&D projects between stakeholders in science and industry, and sponsorship of young scientists. This is precisely what networked structures and the competency networks discussed early support.

\section{References}

[1] Braun, K., \& Erb, R.W., Bionik - Zukunftswissenschaft mit Vergangenheit. Spektrum Bionik - Vorbild Natur in Leben und Technik, Wissen Media Verlag: Gütersloh/München, pp. 12-21, 2008.

[2] www.biokon.net

[3] www.biokon-international.com 